\title{
РАЗРАБОТКА НЕЙРОСЕТЕВОЙ МОДЕЛИ ДЛЯ ДИАГНОСТИРОВАНИЯ БОЛЕЗНЕЙ ЖЕЛУДОЧНО-КИШЕЧНОГО ТРАКТА У СОБАК
}

\section{DEVELOPMENT OF A NEURAL NETWORK MODEL FOR DIAGNOSING DISEASES GASTROINTESTINAL TRACT IN DOGS}

\author{
E. Tolstova \\ A. Ormeli \\ M. Bolshelapov \\ A. Selyutin
}

Summary. The article provides a solution to the problem of diagnosing diseases of the gastrointestinal tract in dogs. This task is relevant for expert veterinarians. To solve this problem, a neural network model of a multi-layer perceptron was developed, which allows classifying diseases depending on incoming input parameters, and a web interface was created for the trained model. The developed interface allows you to interact with the system in the most comfortable way.

Object: development of a multi-layer perceptron neural network model for classification of diseases of the gastrointestinal tract in dogs.

Methods: general theoretical - analysis of special literature, study of statistical data; mathematical — network modeling, programming, visualization.

Findings: a system for diagnosing diseases of the gastrointestinal tract in dogs was developed. The system has a user-friendly web interface.

Conclusions: the developed system can be used by veterinarians when there are difficulties in diagnosing a specific disease of the gastrointestinal tract in dogs.

Keywords: gastritis, pancreatitis, stomach ulcer, Flask, multi-layer perceptron.
Толстова Елизавета Антоновна Саратовский Государственный Аграрный Университет им Н.И. Вавилова г. Саратов liza.tolstova.2014@mail.ru

Ормели Александр Федорович

Саратовский Государственный Технический Университет им. Гагарина Ю. А., г. Саратов ormelialex@gmail.com

Большелапов Михаил Александрович Саратовский Государственный Технический Университет им. Гагарина Ю. А., г. Саратов mihan3110@yandex.ru

Селютин Александр Дмитриевич Саратовский Государственный Технический университет им. Гагарина Ю. А., г. Саратов cool.selutin99@yandex.ru

Аннотация. В статье приведено решение проблемы диагностики заболеваний желудочно-кишечного тракта у собак. Данная задача является актуальной для экспертов ветеринаров. Для решения стоящей проблемы разработана нейросетевая модель многослойного перцептрона, позволяющая классифицировать заболевания в зависимости от поступающих входных параметров, а также, создан веб-интерфейс для обученной модели. Разработанный интерфейс позволяет взаимодействовать с системой максимально комфортным способом.

Цель: разработка нейросетевой модели многослойного перцептрона для классификации болезней желудочно-кишечного тракта у собак.

Методы: общетеоретические — анализ специальной литературы, изучение статистических данных; математические - сетевое моделирование, программирование, визуализация.

Результаты: была разработана система диагностики болезней желудочно-кишечного тракта у собак. Система имеет удобный пользовательский веб-интерфейс.

Выводы: разработанная система может использоваться ветеринарами при возникновении трудностей диагностики конкретного заболевания желудочно-кишечного тракта у собак.

Ключевые слова: гастрит, панкреатит, язва желудка, Flask, многослойный перцептрон. 


\section{Введение}

C каждым годом качество ветеринарной медицины повышается, формируются новые подходы, появляются новые программы, с помощью которых заболевания диагностируются быстрее, чем в случае традиционной работы ветеринаров. По статистике самыми распространенными заболеваниями среди животных, являются заболевания желудочно-кишечного тракта и дыхательной системы, так как они контактируют с внешней средой [3, с. 982].

В связи с этим, возникает необходимость создания нейросетевой модели многослойного перцептрона для классификации болезней желудочно-кишечного тракта у собак, которая на основании описанных симптомов (анализов) предложит несколько вариантов заболеваний и поможет поставить наиболее вероятный диагноз ветеринарному специалисту.

\section{Материалы \\ и мето $\Delta$ ы}

В связи с трудностью определения патологий желудочно-кишечного тракта у собак, даже после ряда обследований, необходимо диагностировать заболевания по ряду анализов. Разрабатываемая система должна давать ответ, в процентном соотношении, на вопрос о наличии конкретной патологии желудочно-кишечного тракта у обследуемой собаки [4, с. 482]. Также информационная система должна обладать удобным и понятным интерфейсом.

Целью текущей работы является разработка нейросетевой модели многослойного перцептрона для классификации болезней желудочно-кишечного тракта по ряду входных параметров. Описанный выше вид нейросетевой архитектуры лучше всего подходит для анализа, дифференциации и классификации простых типов данных. Будет необходимо обработать полученные данные анализов собак, привести их к одной ранговой шкале. Также, потребуется верно подобрать оптимальное количество слоев для модели, чтобы она обладала достаточной точностью и верно дифференцировала входящие данные [2, с. 382]. Помимо этого, необходимо готовой и обученной модели перцептрона придать понятный пользовательский интерфейс. Разработка модели будет происходить с использованием языка программирования Python версии 3.6.6. Будут использованы такие библиотеки для машинного обучения как Keras, а в качестве основы для наиболее главных операций будет использоваться библиотека от Google под названием TensorFlow. Веб-интерфейс будет разработан на фреймворке для создания веб-приложений Flask.

\section{^итературный обзор}

Сегодня существует большое разнообразие нейросетевых архитектур, которые способны выполнять разные задачи. Также существуют разнообразные методы обучения нейросетей, например, с учителем и без. В обучении с учителем множество данных, над которым происходит работа модели, заранее содержит ответ, который должна выдавать система после завершения процесса обучения, но уже на новых данных согласно исследованиям Розенблатта. При обучении без учителя система должна прийти к балансу многократным повторением, благодаря более однозначному выявлению с каждой итерацией различных зависимостей [1, с. 282]. Для текущей задачи, связанной с дифференцированием массивов данных, оптимально будет использовать архитектуру многолослойного перцептрона, согласно работам У. Маккалока и У. Питтса. При разработке будет использоваться фреймворк Keras, содержащий в своей основе фреймворк для TensorFlow. Фреймворк Keras содержит в себе множество алгоритмов для обучения нейросетевых моделей, а также позволяет очень удобно конфигурировать разрабатываемую модель. Благодаря абстракции, которую обеспечивает фреймворк разработка нейросетевой модели будет проходить безболезненно.

Из открытых источников были собраны и обработаны данные об анализах собак с конкретным заболеванием желудочно-кишечного тракта. Данные группировались по патологии. В итоге, было сформировано 4 результирующих подвыборки с патологиями гастрита, язвы желудка, непроходимости кишечника и панкреатита [6, с. 187].

Количество записей, которые удалось корректно обработать, равняется 1353. Этого будет достаточно, чтобы обучить модель, с достаточно высокой точностью и классифицировать заболевания желудочно-кишечного тракта у собак. Формат, в котором хранятся полученные данные о собаках - csv файл [5, с. 382]. Все записи содержат метку класса, то есть обучение модели происходит с учителем, ответы будут оптимизировать веса нейросети.

Для максимально эффективного обучения модели необходимо разделить имеющийся набор данных на 3 выборки по которым будет проводиться дальнейшее обучение и проверка. Был написан скрипт, который размещает в случайном порядке записи по 3 файлам: файл для обучения модели, файл для валидации модели, а также файл проверки модели на явление переобучения. После исполнения скрипта и распределения записей по файлам в файле для обучения модели оказалось 859 записей, в валидационном файле находится 264 записей и в файле для проверки 230 строк со сведениями об анализах и диагностированных патологиях желудочно-кишечного тракта собак. 
Диагностика

Ha гnaвryo

Введите возраст собаки

Укажите пол собаки

Мужской Ю Женский

Введите температуру тела

Собака находится в состоянии беременности?

Да • Нет

Введите массу тела собаки

Укажите рост собаки

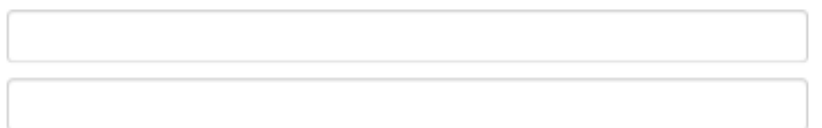

Количество тромбоцитов

Значение скорости оседания эритроцитов

Количество нейтрофилов

Количество лимфоцитов

Значение гемоглобина

Показатель щелочной фосфотазы

Уровень креатинина

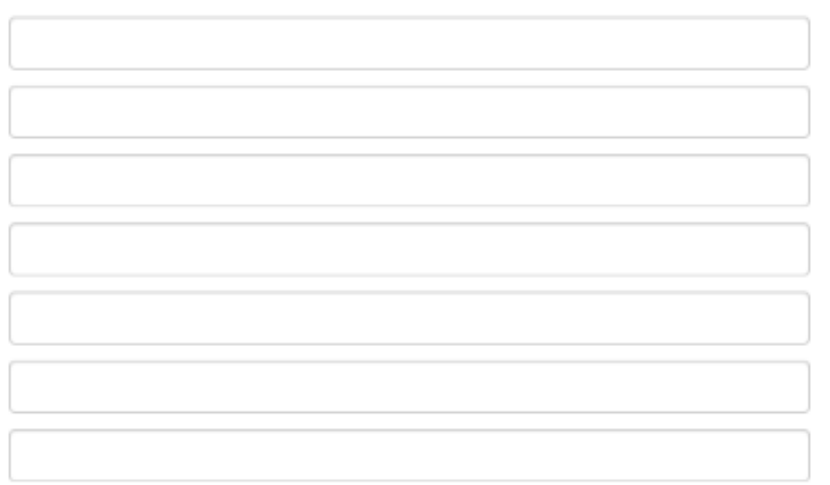

Рис. 1. Веб-интерфейс информационной системы

После распределения записей необходимо заняться разработкой модели. Первым делом следует верно добавить слои и активационную функцию. Благодаря фреймворку Keras сделать это очень просто. Было добавлено 2 промежуточных слоя. Перцептрон представляет собой сужающуюся модель от входного слоя с описанными выше параметрами до 1 нейрона, с предсказанием по поводу патологии желудочно-кишечного тракта на выходном слое. Обучение происходит минибатчами. Это означает, что обучающее множество разбивается на небольшие кортежи по 20 строк, а общие для всей модели веса корректируются в конце итерации. Всего таких итераций обучения 400.

Обучение модели на рабочей станции с процессором Intel Core i7-6700HQ, 16 GB RAM, видеоадаптером NVIDIA GeForce GTX 960M заняло 15 минут. После этого модель была сохранена в формате h5 для дальнейшего использования. На обучающем множестве была достиг- нута точность 95\%. На тестовом множестве точность составила 92\%. Далее необходимо создать удобный интерфейс пользователя, чтобы модель было удобно использовать.

\section{Результаты}

Для разработанной модели необходимо создать веб-интерфейс в виду его чрезмерного удобства и универсальности использования.

Интерфейс должен обладать следующим минимальным функционалом: возможность внести анализы собаки (после обследования), получить результат о конкретной диагностированной системой патологии желудочно-кишечного тракта.

Для разработки был выбран фреймворк Flask. Данная технология обладает рядом преимуществ, например, 
удобнейший шаблонизатор Jinja 2, а также замечательный маршрутизатор Werkzeug. Все эти преимущества позволят быстро и качественно разработать веб-интерфейс.

Необходимо разработать функцию, которая будет принимать, поступившие данные от веб-формы и обрабатывать результат с помощью нейросетевой модели. Потребуется выделить отдельный поток для данной функции, так как действие по обработке и классификации томограммы должно обрабатываться параллельно с НТТР-запросом.

На Рисунке 1, который представлен ниже, изображён интерфейс готовой информационной системы, опубликованной на хостинге Heroku:

\section{Обсужление}

Полученные результаты могут использоваться в работе врачей-ветеринаров при возникновении трудностей, связанных с диагностикой заболеваний желудочно-кишечного тракта у собак. Успешно диа- гностируются такие болезни как: гастрит, язва желудка, непроходимость кишечника и панкреатит. Результаты работы информационной системы проверены практически.

\section{Б^агоАарности}

Авторы выражают признательность Правительству Саратовской области за оказанную помощь при написании настоящей статьи.

\section{Зак^ючение}

В ходе работы была разработана нейросетевая модель многослойного перцептрона, выполняющая функцию диагностики заболеваний желудочно-кишечного тракта, в частности гастрита, язвы желудка, непроходимости кишечника и панкреатита у собак. К разработанной модели был создан веб-интерфейс.

Разработанная система может применяться врачами-ветеринарами в случае возникновения неоднозначностей и трудностей при диагностировании патологий.

\section{ЛИТЕРАТУРА}

1. Васильев А. Н. Принципы и техника нейросетевого моделирования / А. Н. Васильев, Д. А. Тархов. — Москва: СПб. [и др.]: Питер, 2014.

2. Круглов В. В. Искусственные нейронные сети. Теория и практика / В. В. Круглов, В. В. Борисов. — М.: Горячая линия - Телеком, 2002.

3. Лапиков Сергей Никитович Паразитарные болезни собак / Лапиков Сергей Никитович.— М.: Аквариум, 2009.

4. Неонатология и болезни новорожденных собак. Монография. - Москва: ИЛ, 2016.

5. Основы нейрокибернетики.-Москва: СПб. [и др.]: Питер, 2015.

6. Сидоркин В.А. Болезни собак / В. А. Сидоркин.-М.: Аквариум, 2011.

(ㄷ Толстова Елизавета Антоновна ( liza.tolstova.2014@mail.ru ), Ормели Александр Федорович ( ormelialex@gmail.com ), Большелапов Михаил Александрович ( mihan3110@yandex.ru ), Селютин Александр Дмитриевич ( cool.selutin99@yandex.ru ).

Журнал «Современная наука: актуальные проблемы теории и практики» 